rbe

\title{
Reformas Tributária e Previdenciária e a Economia Brasileira no Longo Prazo*
}

\author{
Vladimir Kühl Teles ${ }^{\dagger}$, Joaquim P. Andrade
}

Sumário:1. Introdução; 2. O Modelo; 3. Calibração; 4. Resultados das Simulações; 5. Comentários Finais; Palavras-chave: Crescimento Econômico Endógeno; Brasil; Reforma Previdenciária; Reforma Tributária. Códigos JEL: E60; H52; H55.

O presente artigo busca simular o efeito das reformas tributária e previdenciária sobre variáveis chave da economia brasileira no longo prazo. Para tanto, um modelo de equilíbrio geral computável de gerações superpostas sob a especificação de um modelo de crescimento endógeno à la Lucas (1988) é utilizado. Dentre os principais resultados pode-se destacar que as reformas por si só não são capazes de melhorar a performance de variáveis como taxa de juros e crescimento no longo-prazo, entretanto, ao criar oportunidades para diminuir a dívida pública e aumentar o investimento em educação, tornam-se fundamentais para uma melhoria em tais variáveis.

The paper simulates the effects of tax and social security reforms on the main variables of the Brazilian Economy in the long run. For that matter a computable general equilibrium model of overlapping generations à la Lucas (1988) is employed. The main results are that the reforms in themselves are not capable of affecting the performance of the interest rate and the long run growth rate of the economy. However, as long as they make it possible to reduce the public debt and to increase the investment in education, they become important for the improvement of those variables.

\footnotetext{
* Os autores agradecem os comentários e as sugestões feitas por Jorge Arbache, Bernardo Mueller, Adolfo Sachsida, Samuel Pessôa, Roberto Ellery, David De la Croix e Frédéric Docquier. Qualquer erro remanescente é de inteira responsabilidade dos autores.

†Fundação Getúlio Vargas, Escola de Economia de São Paulo (EESP/FGV). Rua Itapeva 474, 12o. andar, 01332-000, (11)3281-3378, (11)3281-3357, São Paulo - SP. Fundação Getúlio Vargas. Escola de Economia de São Paulo (EESP-FGV). vkteles@fgvsp.br.

¥Universidade de Brasília. Departamento de Economia. jandrade@unb.br.
} 


\section{INTRODUÇÃO}

Os anos recentes tem testemunhado uma enorme divulgação da idéia de que as reformas tributária e previdenciária são necessárias para a geração de crescimento no longo-prazo. Em especial no caso brasileiro um extensivo debate tem sido posto em prática com argumentos a favor e contra as reformas. Tal debate surge tanto no âmbito acadêmico, quanto no âmbito da sociedade como um todo. A pergunta iminente que é levantada nesse debate é: as reformas irão levar a economia brasileira a uma trajetória de crescimento auto-sustentável?

Uma vasta literatura fornece respostas muitas vezes contraditórias sobre tal questão. Sob a reforma tributária, a idéia que um aumento da tributação do consumo para diminuir a tributação sobre o capital, mantendo a receita do governo constante, tende a diminuir a taxa de juros e, por conseqüência, elevar a acumulação de capital é defendida por uma série de estudos como Fullerton et alii (1983), Seidman (1984), Toder (1995), Hall (1996), Hall e Rabushka (1996). Ainda em defesa a reforma tributária, Heer e Trade (2003) demonstram que ela teria um efeito positivo sobre o bem-estar e sobre o nível de emprego da economia, além de aumentar o nível de poupança e Makdissi e Wodon (2002) argumentam que a reforma tributária seria um instrumento capaz de reduzir o nível de pobreza dos países.

Por outro lado, Pechman (1987) argumenta que um aumento do tributo sobre o consumo causaria uma distorção sobre a decisão do consumidor, e como conseqüência, perdas de eficiência. Ao mesmo tempo, Feldstein (1995) defende que um aumento do tributo sobre o consumo com uma diminuição sobre a renda geraria um aumento da taxa de juros em vez de uma queda e Lin (1999) demonstra que em uma economia aberta, os efeitos da reforma tributária sobre as taxas de juros e sobre a acumulação de capital podem ser benéficos ou perversos, dependendo das regras institucionais do sistema tributário. Por fim, Strulik (2003) argumenta ainda que os efeitos da reforma tributária tendem a ser insignificantes sobre o crescimento econômico.

A reforma da previdência, por sua vez, é considerada como fundamental especialmente por causa da insustentabilidade do sistema atual diante da mudança demográfica que o Brasil se depara, bem como a problemas econômicos como o desemprego estrutural e ainda pelos problemas fiscais debelados pela previdência (ver Oliveira et alii (1997)).

Sob um ponto de vista teórico a base da defesa da reforma previdenciária encontra-se em Auerbach e Kotlikoff (1987), que argumenta que uma mudança para um sistema de capitalização elevaria a taxa de poupança da economia, e, por conseguinte, a um aumento da acumulação do estoque de capital. Tal argumento fortemente difundido tem sido reavaliado sob a hipótese da existência de risco ${ }^{1}$. Além disso, certos aspectos das reformas previdenciárias tem sido questionados, fundamentalmente a partir do artigo de Orszag e Orszag e Stiglitz (2001), que argumenta que as justificativas das reformas previdenciárias tem girado em torno de muitos "mitos" que não seriam sustentados na teoria nem na prática. Tal artigo estimulou o desenvolvimentos de outros artigos que corroboram e/ou expandem o seu argumento (e.g. Mesa-Lago (2002)).

Por fim, ao mesmo tempo que esses argumentos são construídos, o desenvolvimento recente dos modelos de crescimento endógeno abriu novas perspectivas para o debate teórico acerca dos efeitos das reformas sobre o crescimento de longo prazo. Diversos autores tem então usado as especificações dos modelos de crescimento endógeno para averiguar os efeitos das reformas sobre o crescimento. No que concerne a reforma tributária, por exemplo, Lucas (1990) prevê apenas um pequeno impacto sobre o crescimento, enquanto que Jones et alii (1993) encontra fortes respostas. Ao mesmo tempo, Stokey e Rebelo (1995) argumenta que fortes respostas não seriam compatíveis com a experiência pós-guerra dos Estados Unidos.

Nesse respeito, o presente artigo busca fornecer uma resposta formal a essa questão para a economia brasileira a partir de simulações construídas à base de um modelo de equilíbrio geral computável. Para o Brasil diversos estudos realizaram simulações dos efeitos das reformas sobre a performance da

${ }^{1}$ Especialmente para o caso brasileiro tal questionamento entra em voga (ver de Ellery e Bugarin (2003) 
economia, como Barreto (1995), Barreto (1997), Miranda (1997), Lannes (1999), Barreto e de Oliveira (2001) e de Ellery e Bugarin (2003), para a reforma previdenciária, e de Araújo e Leon (2003) e Menezes e Barreto (1999), para a reforma tributária. O presente artigo busca fornecer uma contribuição nesta linha de pesquisa usando como paradigma um modelo de crescimento endógeno à la Lucas (1988). Assim, a contribuição do presente trabalho se direciona não apenas no fato de se usar um paradigma alternativo para as simulações como também pelo fato de vislumbrar os efeitos das reformas sobre a acumulação de capital humano, uma vez que este tem sido considerado por um número significativo de estudos como a força motriz do crescimento econômico no longo-prazo ${ }^{2}$, e que a relação entre as reformas e a acumulação de capital humano pode não ser desprezível (ver Casarico (1998)).

Assim, a próxima seção destina-se a apresentar o modelo que será utilizado para se conduzir as simulações, bem como a definição de equilíbrio resultante deste. A seção 3 se deterá às calibrações dos parâmetros a serem feitas para o caso brasileiro, e a seção 4 à apresentação dos resultados. A última seção destina-se aos comentários finais.

\section{O MODELO}

Nesta seção será apresentado o modelo a ser utilizado para analisar o impacto das reformas tributária e previdenciária sobre o crescimento econômico de longo-prazo e sobre variáveis chave da economia como taxa de juros e tempo gasto pelas famílias na acumulação de capital humano. O modelo foi desenvolvido por Bouzahzah et alii (2002) e consiste em uma adequação do modelo de crescimento endógeno de Lucas (1988).

O modelo é construído com base em uma economia fechada com gerações superpostas de adultos. Os agentes são homogêneos entre as gerações e vivem por seis períodos de vida, cada um destes representando dez anos (a primeira geração inicia-se com 18 anos, terminando com 28, onde se inicia a segunda geração e assim por diante). O tamanho da geração $t$ segue um padrão de crescimento populacional dado por,

$$
N_{t}=m_{t} N_{t-1}
$$

onde $m_{t}$ é um mais a taxa de crescimento populacional entre as gerações $t-1$ e $t$.

é considerado um modelo de crescimento endógeno à la Lucas. No primeiro período de vida os agentes jovens tem um estoque de capital humano inerente e tem a possibilidade de aumentá-lo ao devotar uma parte do seu tempo à acumulação de capital humano. Em adição dois tipos de externalidades são provocados pelo capital humano. O primeiro é uma externalidade intergerencial onde o capital humano investido nos jovens no tempo $t$ é parcialmente transmitido a geração seguinte. E o segundo é uma externalidade intratemporal onde um aumento da média de capital humano no tempo $t$ eleva a produtividade contemporânea de cada fator de produção.

\subsection{O Comportamento das Famílias}

$O$ agente representativo maximiza no momento $t$ uma função utilidade intertemporal que depende da seqüência de consumo ao longo de sua vida. Ele assume uma função utilidade aditivamente separável na forma de uma CES como segue,

$$
U_{t}=\sum_{t=1}^{6} \gamma^{j-1} \frac{\left[c_{t+j-1}^{j}\right]^{1-1 / \sigma}-1}{1-1 / \sigma}
$$

\footnotetext{
${ }^{2}$ para uma survey detalhada sobre a relação ente capital humano e crescimento de longo prazo ver Sianesi e Reenen (2003)
} 
onde $j$ é o j-ésimo período de vida, $\gamma \in(0,1)$ é o peso relativo dado à utilidade do próximo período (uma medida de preferência temporal) e $\sigma \in \Re_{+}$mede a elasticidade de substituição intertemporal.

Assumindo mercado de crédito perfeito a restrição orçamentária intertemporal requer igualdade entre os valores descontados das rendas e dos gastos do tempo de vida. Se $r_{t}+1$ denota a taxa de juros entre as datas $t$ e $t+1$, então a taxa de desconto aplicada à renda e ao gasto de $t+j$ é dada por,

$$
R_{t}^{t+j}=\prod_{s=t+1}^{t+j} \frac{1}{1+r_{s}}
$$

O valor presente dos gastos é dado por,

$$
E_{t}=\sum_{j=1}^{6} c_{t+j-1}^{j}\left(1+\tau_{t+j-1}^{c}\right) R_{t}^{t+j}
$$

enquanto que o valor presente do ciclo de vida da renda é dado por

$$
W_{t}=\sum_{t=1}^{6}\left(\left(1-\tau_{t}^{w}\right) w_{t+j-1} l_{t+j-1}^{j} h_{t+j-1}^{j}+T_{t+j-1}^{j}\right) R_{t}^{t+j}
$$

onde $\tau_{t}^{c}$ é a taxa de tributação do consumo em $t$, e $\tau_{t}^{w}$ é a taxa proporcional da tributação sobre a renda do trabalho, $w_{t}$ é o salário bruto por unidade de trabalho efetivo em $t, l_{t+j-1}^{j}$ mede a oferta de trabalho da geração $t$ à idade $j, h_{t+j-1}^{j}$ é o nível de educação à idade $j$ e $T_{t+j-1}^{j}$ equivale às transferências públicas recebidas à idade $j$.

Para cada membro da geração $t$ a seqüência de oferta de trabalho é dada por,

$$
\left(l_{t}^{1}, l_{t+1}^{2}, l_{t+2}^{3}, l_{t+3}^{4}, l_{t+4}^{5}, l_{t+5}^{6}\right) \equiv\left(1-e_{t}, 1,1,1,1-\alpha_{t+4}, 0\right)
$$

onde $e_{t}$ é o tempo investido em educação no primeiro período da vida e $\alpha_{t+4}\left(\alpha_{t+4} \in(0,1)\right)$ é a parcela de tempo que a quarta geração passa aposentada. Assim, a oferta de trabalho é determinada exogenamente com excessão do primeiro período da vida onde o agente pode escolher o tempo gasto com o acúmulo de capital humano.

Seguindo Lucas (1988) o tempo gasto em acumulação de capital humano eleva a eficiência do trabalho. A seqüência de capital humano para a geração nascida em $t$ é dada endogenamente por

$$
\left(h_{t}^{1}, h_{t+1}^{2}, h_{t+2}^{3}, h_{t+3}^{4}, h_{t+4}^{5}, h_{t+5}^{6}\right) \equiv\left(1, \theta_{2} \varphi\left(e_{t}\right), \theta_{3} \varphi\left(e_{t}\right), \theta_{4} \varphi\left(e_{t}\right), \theta_{5} \varphi\left(e_{t}\right), 0\right) \times h_{t}^{1}
$$

onde $\theta_{i}(i=2, \ldots, 5)$ são os parâmetros medindo a produtividade relativa à idade $i$ comparada à da idade 1. Isso combina os efeitos learning-by-doing bem como a depreciação do capital humano com a idade. A variável $h_{t}^{1}$ mede o estoque de capital humano inerente da geração em $t$. A função $\varphi\left(e_{t}\right)$ é a tecnologia comum de treinamento que transforma investimento educacional em ganhos de eficiência no trabalho, sendo assim um fator crucial na determinação da trajetória do crescimento econômico. A forma funcional de $\varphi$ escolhida é a mais simples possível, sendo dada por,

$$
\varphi\left(e_{t}\right) \equiv 1+\xi e_{t}^{\psi}
$$

onde $\psi \in(0,1)$ e $\xi \in \Re_{+}$são dois parâmetros.

Finalmente, o vetor de transferências públicas por idade é dado por

$$
\left(T_{t}^{1}, T_{t+1}^{2}, T_{t+2}^{3}, T_{t+3}^{4}, T_{t+4}^{5}, T_{t+5}^{6}\right) \equiv\left(\nu_{t} e_{t}\left(1-\tau_{t}^{w}\right) w_{t} h_{t}^{1}, 0,0,0, \alpha_{t+4} p_{t+4}, p_{t+5}\right)
$$

onde $\nu_{t}$ é a taxa de subsídio público sob o custo da educação (uma fração $\nu_{t}$ do custo de oportunidade da educação é coberta por subsídios governamentais) e $p_{t}$ é o benefício alocado aos aposentados em $t$. Os indivíduos que estão trabalhando em tempo parcial recebem uma parcela do benefício. 
A restrição orçamentária intertemporal é dada por,

$$
E_{t} \leq W_{t}
$$

Assim, o problema de maximização do indivíduo que nasce na geração $t$ consiste da maximização da função utilidade sujeita à restrição orçamentária intertemporal e dadas as seqüências de oferta de trabalho, capital humano e transferência pública definidas acima.

Como não consideramos a existência de desutilidade do trabalho, o problema da família é separável, podendo-se determinar a decisão de quanto estudar e de consumo uma de cada vez. Assim, maximizando com respeito ao investimento em educação chegamos ao seguinte resultado,

$$
e_{t}^{*}=\left(\xi \psi \sum_{j=2}^{6} \frac{\left(1-\tau_{t+j-1}^{w}\right) w_{t+j-1} l_{t+j-1}^{j} \theta_{j}}{\left(1-\tau_{t}^{w}\right) w_{t}\left(1-\nu_{t}\right)} R_{t}^{t+j}\right)^{1 /(1-\psi)}
$$

Assim, o investimento em educação cresce com o subsídio público mas decresce com o fluxo salarial corrente, que representa o custo de oportunidade.

Maximizando a utilidade com respeito aos níveis de consumo temos a trajetória do consumo ao longo da vida, dada por,

$$
c_{t+j-1}^{j}=\left[\frac{1+\tau_{t+j-2}^{c}}{1+\tau_{t+j-1}^{c}} \gamma R_{t+j-2}^{t+j-1}\right]^{\sigma} c_{t+j-2}^{j-1}
$$

Substituindo esses resultados na restrição orçamentária teremos o consumo ótimo da geração jovem. As últimas três equações determinam completamente o comportamento ótimo dos agentes da geração $t$. Logo, é possível calcular a dotação ótima de ativos dos agentes ao fim de cada período, obtendo-se,

$$
\begin{aligned}
a_{t+j-1}^{j} & =a_{t+j-2}^{j-1} R_{t+j-2}^{t+j-1}+\left(1-\tau_{t+j-1}^{w}\right) w_{t+j-1} l_{t+j-1}^{j} h_{t+j-1}^{j}+ \\
& +T_{t+j-1}^{j}-c_{t+j-1}^{j}\left(1+\tau_{t+j-1}^{c}\right)
\end{aligned}
$$

onde $a_{t+j-1}^{j}$ denota a dotação de ativos do indivíduo ao fim da idade $j$. é claro que sempre será ótimo morrer sem ativos neste modelo de forma que $a_{t}^{6}$ será sempre igual a zero.

\subsection{O Setor Produtivo}

A cada período de tempo a firma representativa usa trabalho $\left(L_{t}\right.$, em unidade de eficiência) e capital físico $\left(K_{t}\right)$ para produzir o bem final $\left(Y_{t}\right)$. Como em Lucas (1988) é assumida uma função de produção Cob Douglas onde a média do estoque de capital humano por trabalhador $\left(\bar{h}_{t}\right)$ afeta o nível de produtividade agregado:

$$
Y_{t}=A K_{t}^{\beta} L_{t}^{1-\beta} \bar{h}_{t}^{\varepsilon}
$$

onde $\beta$ mede a parcela da renda proveniente do capital, $\varepsilon$ é o parâmetro de externalidade intratemporal, $A$ é um escalar exógeno e $\bar{h}_{t}$ é a média do estoque de capital humano no trabalho.

A oferta de trabalho em unidade de eficiência é dada pela soma dos montantes individuais,

$$
L_{t}=\sum_{j=1}^{5} N_{t-j+1} l_{t}^{j} h_{t}^{j}
$$

logo, a média de capital humano dos agentes é obtida dividindo-se a oferta de trabalho em unidades de eficiência pela quantidade de trabalhadores:

RBE Rio de Janeiro v. 60 n. 1/p. 87-107 Jan Mar 2006 


$$
\bar{h}_{t}=\frac{L_{t}}{\sum_{j=1}^{5} N_{t-j+1} l_{t}^{j}}
$$

A firma comporta-se competitivamente no mercado de fatores. A maximização de lucro requer então a igualdade do produto marginal de cada fator a sua taxa de retorno, ou seja,

$$
\begin{gathered}
w_{t}=A(1-\beta) K_{t}^{\beta} L_{t}^{-\beta} \bar{h}_{t}^{\varepsilon} \\
\delta+r_{t}=A \beta K_{t}^{\beta-1} L_{t}^{1-\beta} \bar{h}_{t}^{\varepsilon}
\end{gathered}
$$

onde $\delta \in(0,1)$ é a taxa de depreciação do capital.

\subsection{Governo}

O governo emite títulos e cobra tributos para financiar seus gastos. Não há tributação sobre a renda de capital no modelo, ou seja, toda a renda dos indivíduos é tributada ou no consumo, ou na própria renda do trabalho. Como o modelo prevê que os retornos dos ativos devem se igualar (Equações 17 e 18) entende-se que uma tributação sobre a renda do capital deve ter um efeito similar à tributação sobre a renda do trabalho, ou seja, do capital humano.

Quatro tipos de gastos públicos são vislumbrados: subsídios à educação, benefícios da aposentadoria, consumo público e juros pagos sobre a dívida pública. A restrição orçamentária do governo pode ser escrita então como segue,

$$
\begin{gathered}
\tau_{t}^{w} w_{t} L_{t}+\tau_{t}^{c} C_{t}+D_{t+1}=N_{t} \nu_{t} e_{t}\left(1-\tau_{t}^{w}\right) w_{t} h_{t}^{1}+\left(N_{t-4} \alpha_{t}+N_{t-5}\right) p_{t}+ \\
+G_{t}+\left(1+r_{t}\right) D_{t}
\end{gathered}
$$

onde $D_{t}$ denota a dívida pública no início do período $t, G_{t}$ e $C_{t}$ medem o consumo público e o consumo privado agregados respectivamente no tempo $t$, podendo ser definidos como,

$$
\begin{aligned}
G_{t} & =\sum_{j=1}^{6} N_{t-j+1} G_{t}^{j} \\
C_{t} & =\sum_{j=1}^{6} N_{t-j+1} c_{t}^{j}
\end{aligned}
$$

onde $G_{t}^{j}$ é o montante de consumo público por agente da idade- $j$ no tempo $t$. é assumido que a razão dos gastos públicos em relação ao estoque de capital humano é constante,

$$
\frac{G_{t}^{j}}{h_{t}^{1(1-\beta+\varepsilon) /(1-\beta)}}=g^{j}
$$

Tal relação leva à constância da parcela dos gastos públicos sobre o produto no longo prazo.

Assim, diversos cenários podem ser simulados ao se realizar ajustes tributários, ajustes nos gastos ou variações na dívida pública. Em especial o presente estudo irá avaliar os efeitos das reformas tributária e previdenciária em curso presente no caso brasileiro. 


\subsection{A Dinâmica da Economia}

A dinâmica desta economia é determinada pela evolução dos estoques de capital físico e humano ao longo do tempo. $O$ estoque de capital físico em $t+1$ é determinado pela quantidade de ativos que as famílias possuem menos a dívida pública.

$$
K_{t+1}=\sum_{j=1}^{5} N_{t-j+1} a_{t}^{j}-D_{t+1}
$$

O capital humano é a força motriz do crescimento econômico no modelo uma vez que ele é o ponto chave da produtividade do modelo. A trajetória do capital humano é determinada pelo estoque de capital humano no período anterior bem como pelo investimento em educação pelos agentes. Assumese além disso que o nível de capital humano inerente aos indivíduos da geração $t$ é igual ao nível de capital humano adquirido pela geração $t-1$ sem levar em consideração contudo a correção específica da idade $\theta_{2}$. Assim temos que,

$$
h_{t}^{1}=h_{t-1}^{1}\left(1+\xi e_{t-1}^{\psi}\right)
$$

\subsection{Equilíbrio Competitivo}

Quando a economia inicia-se em $t=0$, seis gerações estão vivas. A primeira a nascer em $t=0$ irá viver seis períodos. Há então cinco gerações velhas cada uma dotada de ativos e de capital humano. A geração nascida em $t=-5$ atinge a idade $6 \mathrm{em} t=0$ e seu estoque de capital humano é zero. As condições iniciais são então $\left(h_{-1}^{1}, h_{-1}^{2}, h_{-1}^{3}, h_{-1}^{4}, h_{-1}^{5},\right)$ e $\left(a_{-1}^{1}, a_{-1}^{2}, a_{-1}^{3}, a_{-1}^{4}, a_{-1}^{5}\right)$, e o estoque inicial de capital físico $K_{0}$ é obtida por,

$$
K_{0}=\sum_{j=1}^{5} N_{-j} a_{-1}^{j}-D_{0}
$$

onde $D_{0}$ é a dívida pública no período 0 . Assim, o equilíbrio competitivo é definido como segue:

Dadas as condições iniciais $\left(K_{0}, D_{0}\right),\left(a_{-1}^{j}, h_{-1}^{j}\right)_{j=1, \ldots, 5}$, a população exógena $\left(N_{t}\right)_{t \geq 0}$ e as variáveis exógenas de política $d,\left(g^{j}\right)_{j=1, \ldots, 6}$ e $\left(\tau_{t}^{t}, p_{t}, \alpha_{t+4}, \nu_{t}, \tau_{t}^{w}\right)_{t \geq 0}$, um equilíbrio competitivo é caracterizado por,

1. quantidades individuais positivas $\left(c_{t}^{j}, e_{t}, h_{t}^{j}, T_{t}^{j}, G_{t}^{j}, l_{t}^{j}, a_{t}^{j}\right)_{t \geq 0, j=1, \ldots, 6}$,

2. quantidades agregadas positivas $\left(Y_{t}, L_{t}, K_{t+1}, \bar{h}_{t}, G_{t}, C_{t}, D_{t}\right)_{t \geq 0}$, e

3. $\operatorname{preços}\left(w_{t}, r_{t}\right)_{t \geq 0}$

tal que as equações (6), (7), (9)-(25) sejam satisfeitas.

\section{CALIBRAÇÃo}

O modelo descrito na seção anterior nos permitirá simular os efeitos das reformas previdenciária e tributária sobre a economia brasileira. Tal exercício requer a calibração do modelo, ou seja, escolher e calcular os parâmetros do modelo, bem como as variáveis exógenas, a fim de reproduzir satisfatoriamente os valores da economia brasileira. é usualmente argumentado que tal tipo de exercício é limitado pela dificuldade de se obter valores confiáveis para determinados parâmetros. Em especial para modelos de crescimento endógeno, como é o caso do modelo aqui utilizado, tal dificuldade de faz presente, pois muitos parâmetros destes modelos, em especial aqueles relacionados com a educação e seus efeitos 
sobre a economia, não tem um valor consensual apresentado na literatura. Assim, o presente tópico se dividirá em duas etapas. A primeira etapa significa apenas a escolha dos valores dos parâmetros e variáveis que são um consenso para a economia brasileira. A segunda etapa consiste na construção de cenários, onde os parâmetros não-consensuais são alternados.

A primeira etapa é resumida pela tabela 1 . Nós consideramos uma população constante $(m=1)$ de forma que os resultados das taxas de crescimento simuladas sempre estarão em termos per-capita. 0 sistema de seguridade social é parametrizado seguindo os parâmetros para o caso brasileiro propostos por de Ellery e Bugarin (2003), ou seja, a taxa de reposição da previdência social corresponde a $90 \%$ e $\alpha_{t+4}=1$, que corresponde a idade média de aposentadoria de 58 anos $^{3}$. A razão dívida pública - PIB média dos últimos dez anos foi de $41,7 \%$, de forma que pode-se aproximar a razão dívida-PIB de steadystate do Brasil para $40 \%$, sendo este o valor escolhido ${ }^{4}$. 0 tributo sobre consumo segue o calculado por Araújo e Ferreira (1997) de 0,1816.

A participação do capital na renda (capital-share) usado é 0,35. Tal valor situa-se entre os padrões encontrados na literatura internacional como Kydland e Prescott (1982), 0,36; Cooley e Prescott (1995), 0,40; Bouzahzah et alii (2002), 0,29; Lucas (1990), 0,24; ou Auerbach e Kotlikoff (1987), 0,25. Além disso, a taxa calculada com valores do IBGE inclui a remuneração dos trabalhadores conta-própria e dos empregadores como remuneração do capital, superestimando um capital-share calculado a partir de dados oficiais, de forma que não há um número oficial confiável para o Brasil.

Os valores de $\theta_{i}$ seguem uma calibração a partir da função quadrática proposta por Miles (1999) e Bouzahzah et alii (2002), a taxa de depreciação do capital é de $4 \%$ ao ano. $O$ parâmetro de externalidade do capital humano segue Lucas (1988) em 0,1 .

Finalmente a taxa de preferência temporal é de 0,95 que é o valor aproximado que pode ser obtido a partir da taxa de juros observada para a economia brasileira nos últimos anos. A taxa de retorno do capital fisico de longo-prazo para o Brasil é calculada por Gomes et alii (2003) em 6\%, o que leva a uma taxa de preferência temporal de 0,9433, ou, aproximadamente, 0,95 , valor utilizado no texto. Além disso, a taxa de retorno da bolsa de valores americana ao ano entre 1880 e 2000 é de aproximadamente $6 \%$ em seu valor nominal, e $4 \%$ real, sugerindo que a taxa de juros no steady-state é de cerca de $5 \%$ nos EUA. Como demonstrado por Gomes et alii (2003) e Ferreira et alii (2004), a taxa brasileira é a mesma da americana, de forma que este valor pode ser usado para as simulações para o Brasil.

Para os demais valores como elasticidade intertemporal do consumo e dos $g_{i}$ os dados da economia brasileira mostram-se muito pouco confiáveis para uma estimação exata (ver de Ellery e Bugarin (2003)), de forma que os valores escolhidos seguiram o objetivo de se alcançar os resultados mais próximos com os observados na economia. Em particular com relação à elasticidade intertemporal do consumo Issler e Piqueira (2000) encontram um valor bem menor do que o utilizado aqui. Entretanto a adoção de um valor similar ao de Issler e Piqueira implicou em resultados completamente destoantes para as variáveis endógenas e a escolha de um valor menos crível foi crucial para a convergência do modelo.

A segunda etapa deste tópico consiste na construção de três cenários alternativos com parâmetros da função de produção de capital humano distintos. Seguindo Bouzahzah et alii (2002) o parâmetro de retornos decrescentes do investimento em educação $(\psi)$ varia entre 0,1 e 0,3, enquanto que o parâmetro de escala da transformação do capital humano em eficiência $(\xi)$ varia entre 0,23 e 0,31 . Dessa forma tais parâmetros para cada cenário seguem o disposto na tabela 2. Outro parâmetro que é claramente difícil de se estimar é o de taxa de subsídio à educação. Entretanto, o valor base de 0,25 foi escolhido, ou seja, assume-se que $25 \%$ da educação é subsidiada pelo governo. Serão utilizados três valores alternativos para o subsídio à educação: $0,25,0,50$, e 0,75 . Tal exercício foi feito porque não existe nenhuma estimativa confiável de tal valor até onde sabemos. Acima de tudo esta é uma variável de política, de forma

\footnotetext{
${ }^{3}$ O valor usado por de Ellery e Bugarin (2003) é de 57 anos. O valor de 58 anos escolhido é usado por razões óbvias por ser o limite inferior da idade da quarta geração. Essa diferença não tende a alterar substancialmente os resultados a serem apresentados

${ }^{4}$ Os dados utilizados foram anuais pelo IPEADATA, que fornece dados desde 1991. Se utilizarmos dados desde 1991, ou seja, dos últimos treze anos, teremos que a razão dívida-PIB média foi de 40,7\%, de forma que o valor de $40 \%$ utilizado seria mantido.
} 
Tabela 1 - Variáveis Exógenas e Parâmetros Fixos

\begin{tabular}{lll} 
Parâmetros & & \\
\hline Descrição & $A$ & Valor \\
\hline TFP & $\beta$ & 0,35 \\
Capital Share & $\sigma$ & 1,5 \\
Elast. Subst. Inter-Temporal & $\delta$ & 0,04 \\
Depreciação Anual & $\theta_{2}$ & 1,39 \\
Ef. Relativa 28-38 & $\theta_{3}$ & 1,76 \\
Ef. Relativa 38-48 & $\theta_{4}$ & 1,93 \\
Ef. Relativa 48-58 & $\theta_{5}$ & 1,85 \\
Ef. Relativa 58-68 & $\varepsilon$ & 0,1 \\
Par. Externalidade de Lucas & $m$ & 1 \\
Tx de Cresc. Pop. & $\gamma$ & 0,95 \\
Pref. Temporal Anual & $\nu$ & 0,25 \\
Tx. de Subsídio à Educação & & \\
\hline & & \\
Variáveis Exógenas & & Valor \\
\hline Descrição & & $90 \%$ \\
\hline Taxa de Reposição da Previdência & & 1 \\
Tempo de Aposent. entre 58-68 & $\alpha_{t+4}$ & 1 \\
Razão Dív. Públ. / PIB & $d$ & 0,4 \\
Gastos Públicos 18-28 & $g_{1}$ & 1,5 \\
Gastos Públicos 28-38 & $g_{2}$ & 1,5 \\
Gastos Públicos 38-48 & $g_{3}$ & 1,5 \\
Gastos Públicos 48-58 & $g_{4}$ & 1,5 \\
Gastos Públicos 58-68 & $g_{5}$ & 1,5 \\
Gastos Públicos 68-78 & $g_{6}$ & 1,5 \\
Tributo sobre Consumo & $\tau_{c}$ & 0,1816 \\
\hline & &
\end{tabular}

que alterações em seu valor tornam possível avaliar os efeitos de mudanças nos gastos governamentais em educação sobre os resultados do modelo.

Tabela 2 - Calibração sob Diferentes Cenários

\begin{tabular}{cccc}
\hline parâmetros & cenário 1 & cenário 2 & cenário 3 \\
\hline$\psi$ & 0,10 & 0,20 & 0,30 \\
$\xi$ & 0,23 & 0,27 & 0,31 \\
\hline
\end{tabular}

Tabela 3 - Calibração sob Diferentes Cenários: Variáveis Endógenas

\begin{tabular}{lccc}
\hline Valores de Steady-State & Cenário 1 (\%) & Cenário 2 (\%) & Cenário 3 (\%) \\
\hline Tributos sobre Salários & 44,66 & 45,78 & 47,93 \\
Taxa de Crescimento Anual & 1,56 & 1,52 & 1,53 \\
Taxa de Juros Anual & 5,52 & 5,57 & 5,72 \\
Razão Consumo/Produto & 68,62 & 68,82 & 68,98 \\
Razão Investimento/Produto & 18,91 & 18,61 & 18,22 \\
Razão Gastos do Governo/Produto & 12,47 & 12,57 & 12,81 \\
Tempo Devotado à Educação & 4,19 & 8,09 & 11,84 \\
\hline
\end{tabular}

A Tabela 3 nos fornece os resultados de steady-state para as variáveis endógenas calculados a partir do modelo sob os três cenários alternativos. Um primeiro aspecto a ser destacado é que variáveis 
importantes como crescimento e juros parecem não variar substancialmente entre os três cenários. A única variável que sofre tal tipo de mudança drástica é o tempo devotado à educação por parte da primeira geração. Entretanto, como veremos no próximo tópico, os efeitos marginais das reformas e de outras possíveis políticas, como uma política educacional, variam decisivamente a partir da adoção de cada um destes parâmetros.

\section{RESULTADOS DAS SIMULAÇÕES}

Para conduzir as simulações optou-se por utilizar um algoritmo que preserve a natureza não-linear do modelo. Para tanto seguiu-se a metodologia proposta por Boucekkine (1995) para trajetórias de ponto de sela em modelos determinísticos não-lineares e implementada no programa Dynare. O método empregado utiliza o algoritmo de Newton-Raphson além de processos alternativos para resolver e aproximar problemas de ponto de sela e de aproximação em modelos não lineares, onde, a cada linearização, o modelo é resolvido utilizando-se um procedimento específico de triangulation-backsubstitution, perfeitamente adaptado a distribuição de zeros das matrizes Jacobianas envolvidas. Esta metodologia tem como objetivo contornar as dificuldades encontradas em soluções de modelos com expectativas racionais em que métodos recursivos dependem da imposição de valores de contorno. Serve também como método alternativo a técnica de Fair-Taylor que resolve parcialmente este problema. Para maiores detalhes Juillard (1996) e Boucekkine (1995).

O problema de horizonte infinito é aproximado a um horizonte de 30 períodos, uma vez que este mostrou-se suficiente para se obter a convergência ao novo steady-state $e^{5}$. Os resultados serão apresentados em duas subseções complementares, onde na primeira será relizada a análise dos efeitos das reformas sobre o steady-state da economia e na segunda serão observadas as dinâmicas de transição de variáveis endógenas chave a partir destes choques permanentes.

Os experimentos a serem realizados tratam dos efeitos das reformas previdenciária e tributária e de choques na dívida pública ou na política educacional, sobre o crescimento econômico. Sobre este aspecto tem-se como objetivo avaliar a importância das reformas sobre variáveis chave da economia como taxa de juros e crescimento, além do seu efeito fiscal. Por outro lado, uma vez que o efeito fiscal dessas reformas é evidente os exercícios seguintes concernem alternativas de políticas a serem seguidas a partir da folga fiscal proveniente, que envolvem um queda nos gastos para diminuir a dívida ou aumento dos gastos em educação. As perguntas que busca-se uma resposta são: As reformas em si afetam o crescimento de longo-prazo? Quais devem ser as prioridades a partir da folga fiscal proveniente dessas reformas?

\subsection{Análise de Steady-State}

A análise de steady-state das reformas é conduzida para os três cenários discriminados na seção anterior. Ademais, buscou-se realizar simulações para cada reforma de forma individual,e para combinações das reformas em conjunto. Os resultados são apresentados na Tabela 4.

Na Tabela 4 são apresentados os resultados de simulações para seis alternativas de reformas além dos resultados de steady-state antes das reformas para todos os três cenários. Dessa forma a coluna reftrib apresenta o steady-state a ser alcançado pela economia tendo em vista apenas a realização da reforma tributária. A refprev1 é uma alternativa de reforma da previdência obtida por um aumento da idade média de aposentadoria para a idade de 65 anos, ou seja, por uma redução de $\alpha$ para 0,2, enquanto que a refprev2 significa uma redução no valor das aposentadorias em $10 \%$. a refprevtotal significa a aplicação conjuntas das reformas refprev1 e refprev2. Além disso duas alternativas de uso conjunto das reformas foi utilizada, onde em uma se considerou como reforma da previdência apenas o

\footnotetext{
${ }^{5}$ Aumentos no tamanho do período não implicaram em alterações nos resultados
} 


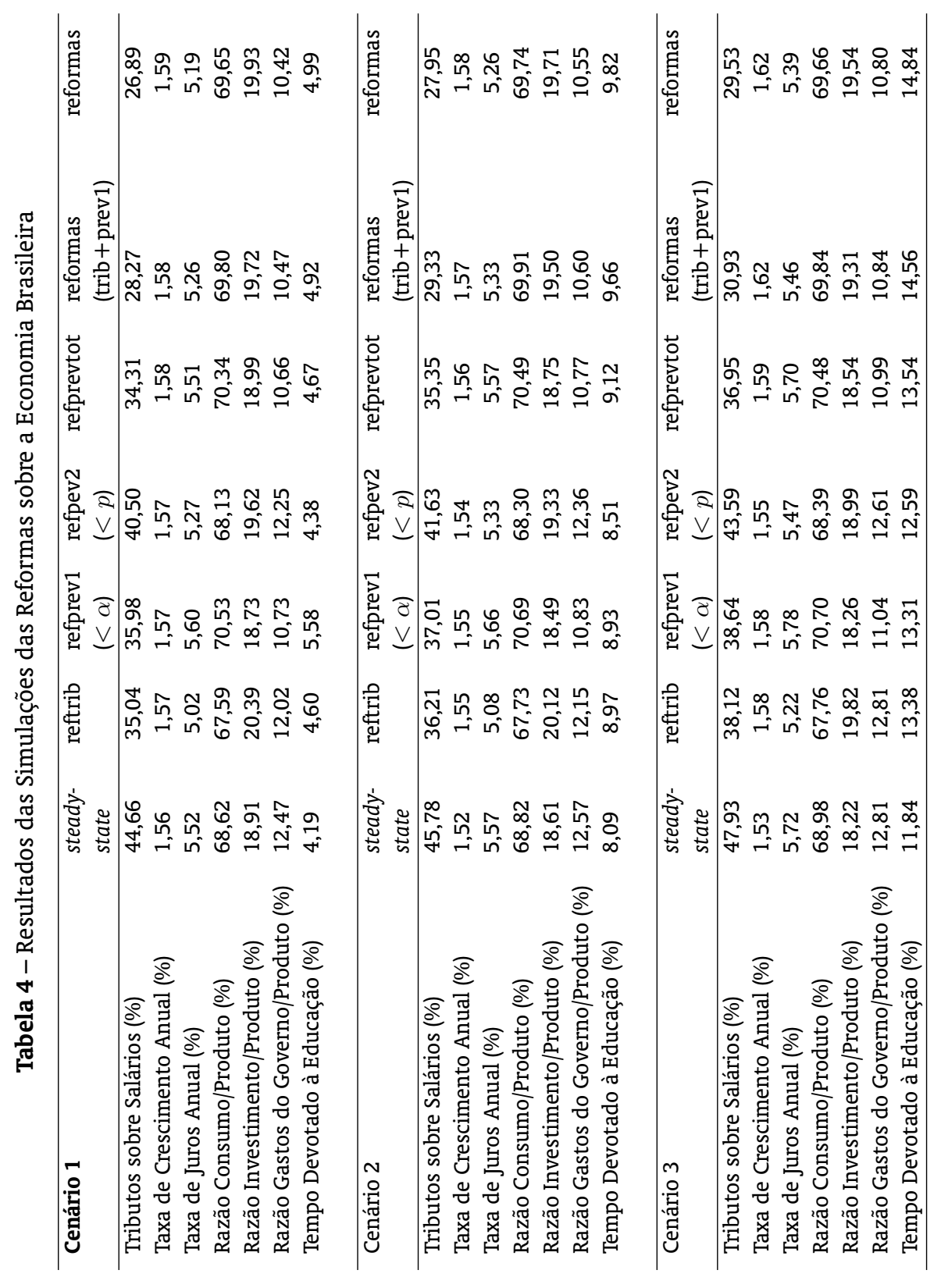


adiamento da aposentadoria (refprev1) junto com a reforma tributária e como outra alternativa a adoção dos três tipos de reformas em conjunto, ou seja, da refprev1, refprev2 e da reforma tributária.

O ponto inicial a ser analisado refere-se ao significado que deve-se dar aos tributos sobre salários. Devemos lembrar que o modelo compreende apenas dois tipos de tributos de forma que, sendo o tributo sobre consumo exógeno, o tributo sobre salários significa o montante total de tributos que deve ser utilizado para se manter a dívida pública constante, entrando no exercício como a variável endógena que fecha o orçamento do governo no steady-state. Dessa forma, o tributo sobre salários como aqui disposto significa uma medida do esforço fiscal que o governo terá de manter para que a dívida pública fique constante. Assim, nos três cenários é possível observar que o valor do tributo sobre salários assume valores entre $44,66 \%$ e $47,93 \%$, ou seja, no steady-state sem reformas a dívida pública só seria mantida constante com um esforço fiscal muito elevado.

A simulação dos efeitos da reforma tributária sobre a economia, apresentada na coluna reftrib reflete um aumento do tributo sobre consumo para 21,84\% seguindo o cálculo de Araújo e Ferreira (1997), resulta nos três cenários em um pequeno efeito sobre o crescimento econômico, mas apresenta um efeito negativo de cerca de meio ponto percentual na taxa de juros de steady-state, o que pode ser considerado um efeito significativo além de um resultado fiscal positivo, vislumbrado pela queda de cerca de $9 \%$ do tributo sobre salários em qualquer um dos três cenários. Dessa forma, o efeito fiscal e a queda dos juros segue o proposto pela literatura que sai em defesa da reforma tributária, mas não o resultado sobre o crescimento e sobre a acumulação de capital, uma vez que a razão investimentoproduto pouco se altera.

Com relação a reforma da previdência é possível se observar que tanto a refprev1, quanto a refprev2 apresentam apenas um pequeno efeito sobre juros e crescimento, porém a refprev1 (aumento da idade de aposentadoria) tem um efeito fiscal de maior magnitude que o da refprev2 (diminuição do valor médio pago de aposentadorias). No que concerne aos efeitos das duas reformas em conjunto é possível notar que o efeito sobre as acumulações de capital físico e humano é insignificante, não ocorrendo o mesmo com o efeito fiscal das reformas.

Duas alternativas de combinações de reformas tributária e previdenciária são simuladas. A diferença entre as duas é a definição da reforma previdenciária, onde a primeira definição exclui a redução do valor médio das aposentadorias, o que não ocorre com o segundo tipo de definição. Tal diferenciação ocorre devido ao argumento que a média do valor das aposentadorias não deve se alterar muito pois esta média já estaria em um valor muito pequeno, de forma que esta redução seria irrealizável. Ao se observar os resultados fica claro que a inserção da redução dos pagamentos médios da aposentadoria não alteram substancialmente os resultados. Como resultados principais observa-se que as reformas se mostram incapazes nestas simulações de alterar significativamente o tempo devotado à educação e a razão investimento-produto, ou seja, as reformas não tendem a afetar a acumulação de capital físico e humano. O resultado sobre a taxa de juros chega a cerca de redução de meio ponto percentual e sobre o crescimento do PIB per capita mostra ser pouco importante. Entretanto, observa-se que as reformas implicariam em um efeito fiscal substancial, ao reduzir o tributo sobre salários necessário para manter a dívida pública constante em quase $20 \%$, ou seja, embora as reformas não levem ao crescimento e a queda dos juros elas possibilitam um atenuado alívio do esforço fiscal para manter o equilíbrio fiscal do governo.

Entretanto a questão que é levantada a partir destes resultados refere-se à utilidade das reformas uma vez que este "alívio" fiscal não significaria aumento do crescimento e tampouco redução significativa dos juros. Neste caso devemos analisar que tipos de políticas poderiam usar deste ganho fiscal para alcançar tais resultados. Para tanto dois tipos de políticas são sugeridos, uma redução da dívida pública de steady-state e um aumento do subsídio à educação. Dessa forma um exercício considerando a economia brasileira pós-reformas (última coluna da tabela 4) o ponto inicial da economia, choques permanentes sobre a dívida pública e sobre o subsídio à educação são implementados de forma a se alcançar os resultados apresentados na Tabela 5.

Os resultados apresentados na tabela 5 deixam claro que os efeitos de um aumento na subsídio à 


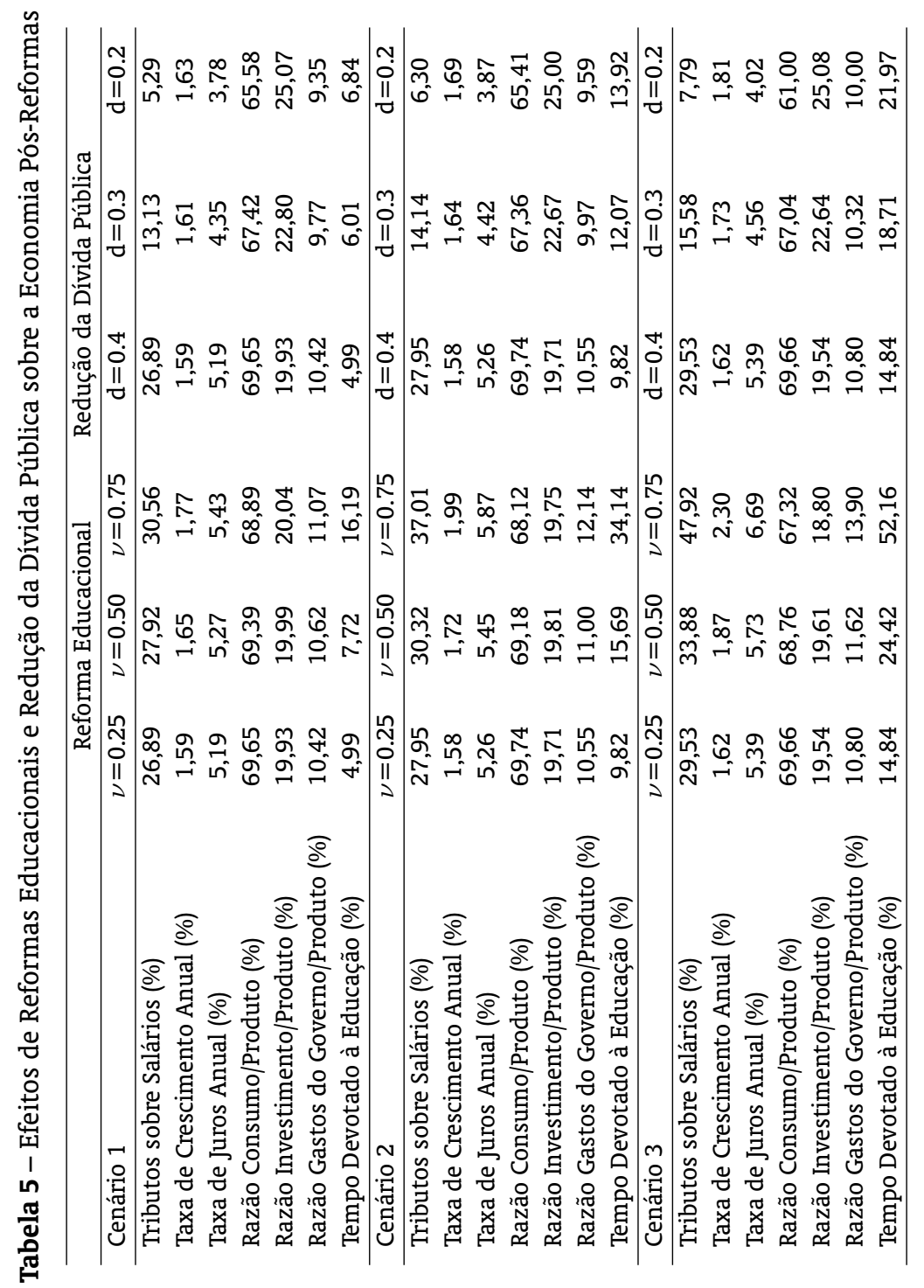


educação variam drasticamente entre os três cenários vislumbrados. Assim, no cenário 3 que considera um efeito mais forte da educação sobre a economia , um aumento do subsídio à educação de $25 \%$ para $75 \%$ iria elevar o tempo devotado à educação da primeira geração de $14,84 \%$ para $52,16 \%$, e da taxa de crescimento anual de $1,62 \%$ para $2,30 \%$. Os resultados seriam muito mais modestos sob os cenários 1 e 2 .

Ao mesmo tempo, um efeito perverso seria observado sobre o esforço fiscal para se manter a dívida pública constante. No cenário 3 o aumento do subsídio à educação - que já é um componente negativo sobre o equilíbrio fiscal - causaria um farto aumento do tempo devotado à educação, que aumenta os gastos do governo uma vez que o governo está subsidiando esse investimento. Assim, o aumento do subsídio à educação causaria uma "reação em cadeia"sobre os gastos públicos elevando o esforço do governo para manter a dívida pública constante em quase $20 \%$ e, consequentemente, a taxa de juros de equilíbrio. Mais uma vez, sob o cenário 1, por exemplo, tais efeitos seriam reduzidos.

No que se refere à redução da dívida pública é possível se observar que esta causaria um efeito extremamente benéfico sobre o tributo sobre salários e sobre a taxa de juros, como seria de se esperar. Ao mesmo tempo, é possível notar que o efeito deste tipo de política sobre o crescimento se daria via acumulação de capital fisico ao invés da acumulação de capital humano, como pode ser observado no aumento substancial na razão investimento-produto.

A relativa constância da taxa de crescimento na maioria dos experimentos deve-se às características do modelo endógeno cujo mecanismo de crescimento depende basicamente do aumento da produtividade do trabalho pela acumulação do capital humano e pela redução da taxa de juros. As reformas tributárias e fiscais alteram muito pouco o tempo dedicado a educação conforme pode se ver na tabela 4, em todos os cenários, ao mesmo tempo as mudanças na taxa de juros são muito pequenas. Por outro lado, após as reformas se políticas de incentivo à acumulação de capital humano e/ou físico forem realizadas, a taxa de crescimento varia substancialmente, como demonstrado nos exercícios relativos à reforma educacional e à redução da dívida pública.

Analisar os efeitos da redução da dívida pública é importante na medida em que o serviço da dívida compromete boa parte do orçamento do governo. Com efeito o resultado mais importante desse experimento é a redução do tributo sobre os salários e da taxa de juros, tendo esta última um efeito importante sobre o crescimento como se pode depreender na tabela 5.

\subsection{Análise da Dinâmica de Transição}

A análise da dinâmica de transição da economia a partir das reformas vislumbradas na seção anterior é realizada sobre três variáveis - taxa de crescimento anual, taxa de juros anual e tributo sobre salários - a partir de cinco alterações na economia que são apresentadas a seguir.

\subsubsection{Reforma Tributária}

Considera-se que nos primeiros dez anos, ou seja, no primeiro período, o tributo sobre salários seja ajustado para o novo patamar determinado pela reforma tributária. Os resultados são apresentados na figura 1.

Os efeitos mais imediatos são aqueles sobre o crescimento uma vez que o choque sobre a decisão de acumular capital humano ocorre de imediato. Por outro lado oserva-se que os ajustes da taxa de juros e do tributo sobre salários são mais suaves e seguem o mesmo padrão, o que indica que esta séries estão fortemente relacionadas no modelo. Neste caso é possível afirmar que, neste modelo, que as variações sobre o esforço fiscal para se manter a dívida pública constante responde de forma co-integrada às variações nos juros. 


\subsubsection{Reforma Previdenciária 1}

A Reforma Previdenciária 1 (refprev1) refere-se a uma mudançana idade de aposentadoria de 58 anos em média para 65 anos, cujo resultado sobre as trajetórias de transição é apresentado na figura 2 .

Neste caso, uma vez que que os gastos com previdência diminuem o tributo sobre salários reduzse bruscamente. Como os agentes se aposentam mais tarde eles precisam de menos poupança para financiar sua velhice, o que eleva os juros momentaneamente. O crescimento dá-se pelo aumento da participação populacional na produção.

\subsubsection{Reforma Previdenciária 2}

A Reforma Previdenciária 2 (refprev2) refere-se a uma queda da relação aposentadoria-salário médio da economia em $10 \%$. Os resultados desta simulação são apresentados na figura 3.

Neste caso se observa que os resultados diferem em sua trajetória da refprev1, porém são muito similares aos resultados da reforma tributária. Nesse sentido os efeitos sobre o crescimento são imediatos, enquanto que os efeitos sobre os juros e sobre a tributação sobre os salários são suaves. As explicações de tais previsões são equivalentes às dadas para os efeitos da reforma tributária.

\subsubsection{Reforma Educacional}

A Reforma Educacional aqui imposta refere-se a um aumento do subsídio à educação de $25 \%$ para $75 \%$ tendo como ponto de partida uma economia pós reformas conforme explicitado na subseção anterior, cujos resultados são apresentados na figura 4.

O resultado imediato deste tipo de reforma é um aumento do tempo devotado à educação, o que implica em um aumento imediato das taxas de crescimento econômico, embora com diferenças drásticas no que concerne à magnitude. Sob o ponto de vista fiscal se observa um crescimento suave dos tributos aos salários necessários para se manter a razão dívida-PIB constante até se alcançar o novo steady-state. Tal comportamento é seguido pela taxa de juros da economia que acompanha o crescimento do tributo sobre salários.

\subsubsection{Redução da Dívida Pública}

Supõe-se que a razão dívida-PIB é reduzida de $40 \%$ para $20 \%$ no primeiro período, ou seja em dez anos, tendo como ponto de partida uma economia pós reformas conforme explicitado na subseção anterior. Os resultados são apresentados na figura 5.

O primeiro efeito desta política é um aumento do tributos sobre salários para se alcançar o objetivo disposto. Uma vez alcançada a meta da dívida o tributo sobre salários reduz-se permanentemente uma vez que o esforço fiscal para se manter a dívida mais baixa agora é menor. Ao mesmo tempo, com uma dívida pública menor a tendência natural de mercado é alcançar uma taxa de juros menor, como pode ser observado no gráfico. Com relação à taxa de crescimento econômico é possível observar um pico por ocasião da redução da dívida, havendo um retorno posterior para uma taxa mais baixa. Isso ocorre porque a redução esperada do tributo sobre salários torna o investimento em educação altamente lucrativo para a primeira geração. Entretanto, no segundo momento a economia converge para uma taxa mais baixa uma vez que o ajuste do tributo sobre salários é obtido.

\section{COMENTÁRIOS FINAIS}

O presente artigo buscou analisar os efeitos das reformas tributária e previdenciária sobre a economia brasileira dentro de um ambiente de crescimento econômico endógeno à la Lucas (1988) a partir de um modelo de equilíbrio geral computável. Sob esse contexto outras reformas como uma reforma educacional e uma redução da dívida pública são simuladas em um cenário pós-reformas. 
Figura 1 - Reforma Tributária

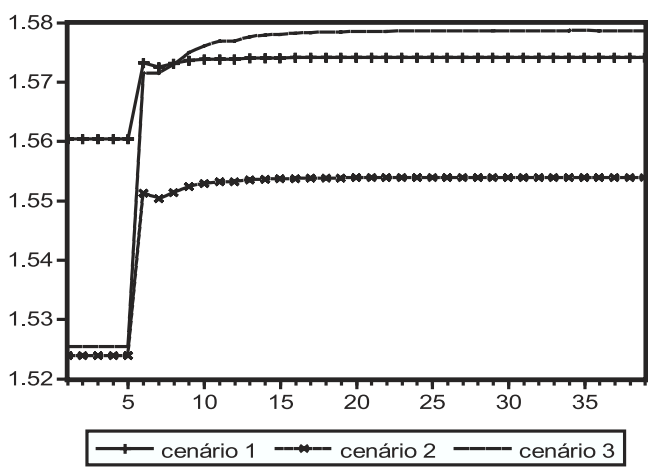

(a) Taxa de Crescimento Anual

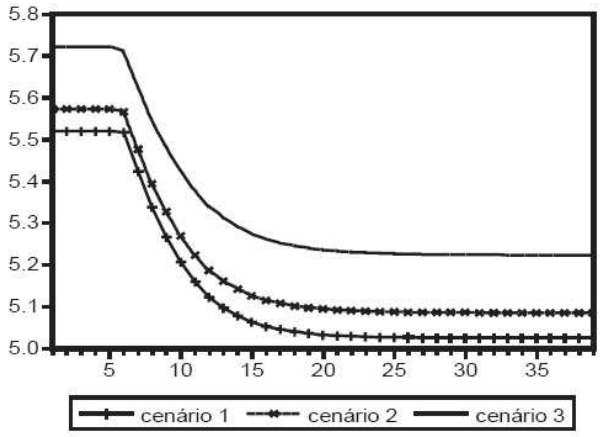

(b) Taxa de Juros Anual

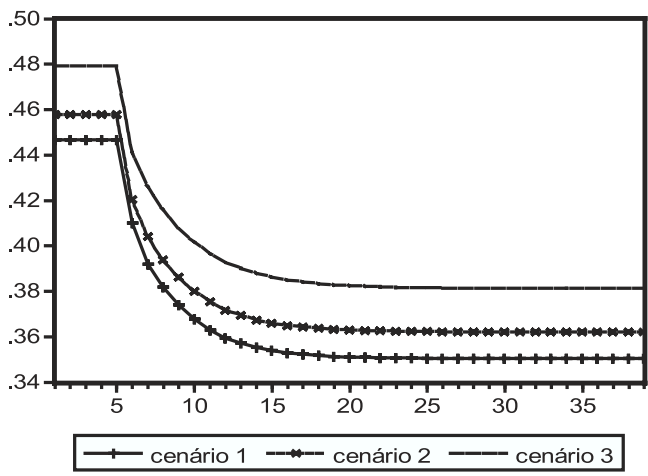

(c) Tributo sobre Salários

Três conclusões centrais puderam ser obtidas a partir deste exercício:

1. As reforma tributária e previdenciária não afetam substancialmente as decisões dos agentes de investirem em capital humano de forma que não afetam o crescimento econômico diretamente, o mesmo ocorrendo com a taxa de juros. Por outro lado, as reformas afetam de forma benéfica o esforço fiscal necessário para se manter uma dívida pública constante.

2. Uma reforma educacional, identificada por um aumento do subsídio dado pelo governo à acumulação de capita humano tem efeitos altamente dependentes dos parâmetros educacionais calibrados. Nesse respeito a taxa de crescimento tende a se elevar, o mesmo ocorrendo, porém, com o esforço fiscal necessário para manter a razão dívida-PIB constante, tendo assim, um efeito sobre o equilíbrio fiscal adverso. Entretanto a magnitude destes dois efeitos depende decisivamente dos parâmetros escolhidos.

3. Uma redução da dívida pública tem um efeito levemente positivo sobre o crescimento. Porém tal efeito ocorre via aumento da acumulação do capital físico resultante da queda dos juros, e não 
Figura 2 - Reforma Providenciária 1

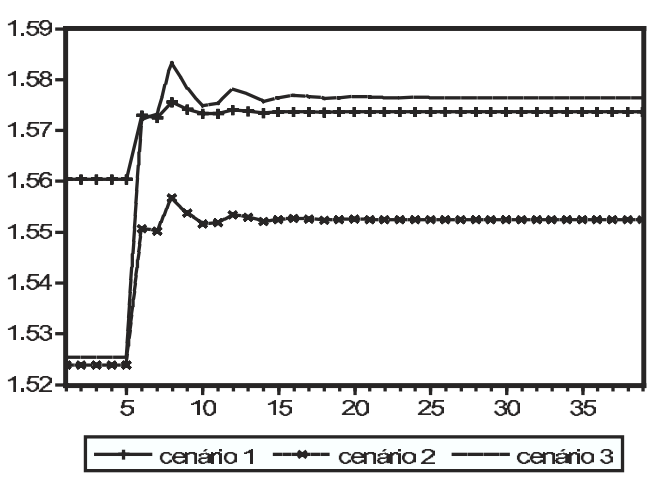

(a) Taxa de Crescimento Econômico Anual

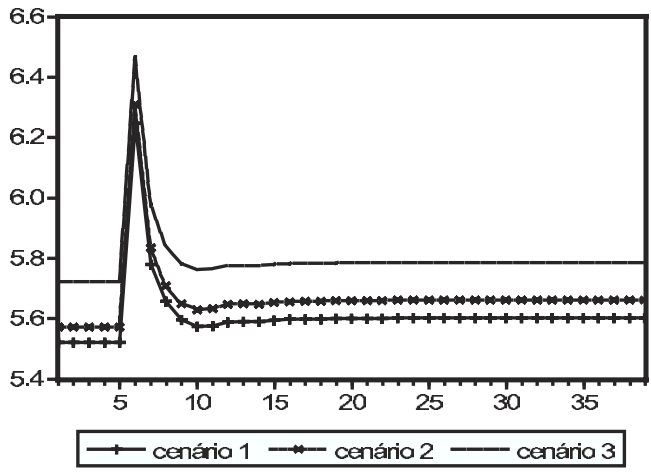

(b) Taxa de Juros Anual

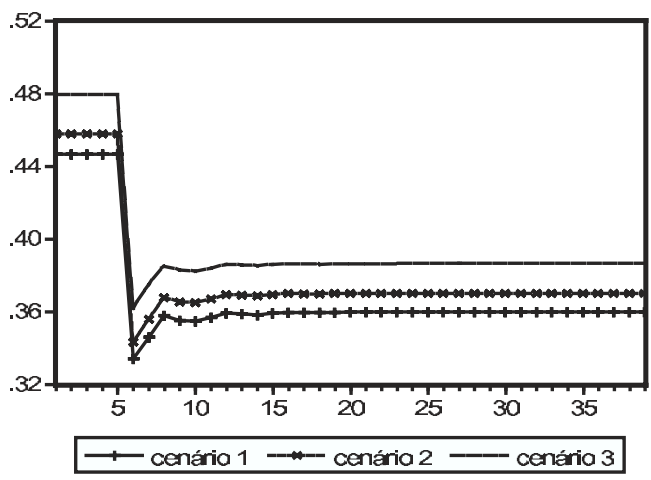

(c) Tributo sobre Salários

via acumulação de capital humano. Com uma dívida menor os juros diminuem o que leva a uma queda do esforço fiscal do governo para manter a dívida constante.

Tais resultados nos fornecem uma lição geral de que as reformas não garantem aumento de crescimento nem tampouco queda nos juros, porém criam condições para que estes eventos tornem-se possíveis. Logo, as reformas seriam uma condição necessária, mas não suficiente para a retomada do crescimento econômico ou para a queda da taxa de juros da economia.

\section{Referências Bibliográficas}

Araújo, C. H. V. \& Ferreira, P. C. (1997). Reforma tributária no brasil: Efeitos alocativos e impactos no bem-estar. Ensaios Econômicos da EPGE no 316.

Auerbach, A. J. \& Kotlikoff, L. J. (1987). Dynamic Fiscal Policy. Cambridge University Press, Cambridge. 
Figura 3 - Reforma Providenciária 2

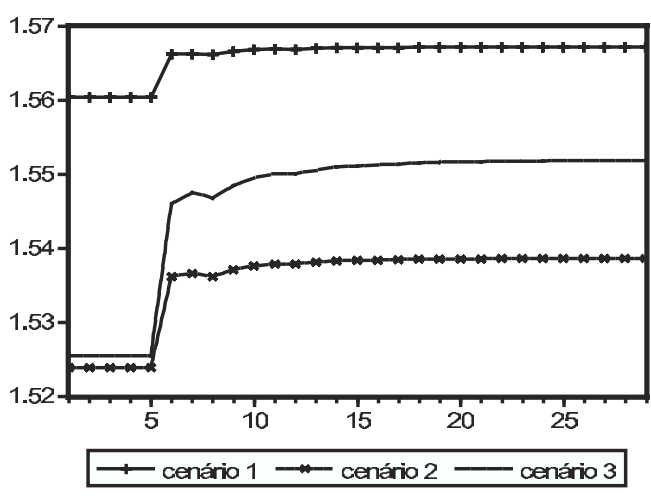

(a) Taxa de Crescimento Econômico Anual

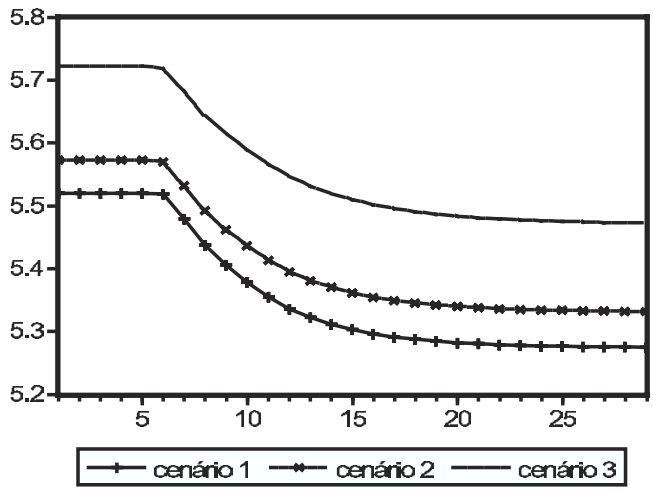

(b) Taxa de Juros Anual

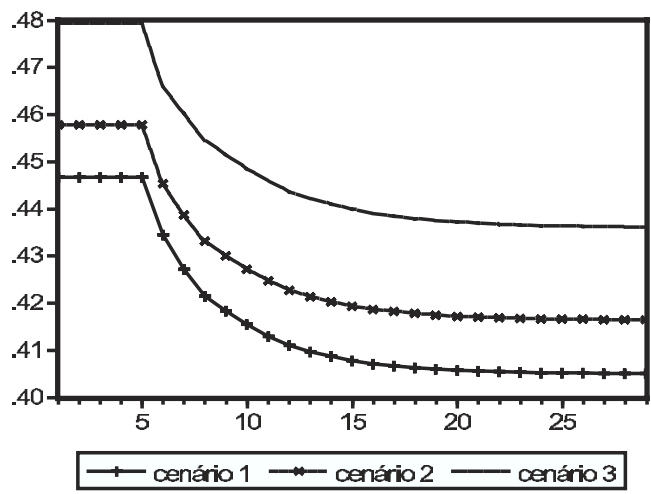

(c) Tributo sobre Salários

Barreto, F. A. F. D. (1995). Aplicação de um modelo de gerações superpostas para a reforma da previdência no brasil: Uma análise de sensibilidade no estado estacionário. In Anais do XVII Encontro Brasileiro de Econometria, Salvador. Sociedade Brasileira de Econometria.

Barreto, F. A. F. D. (1997). Três ensaios sobre reforma de sistemas previdenciários. PhD thesis, Escola de Pós-Graduação em Economia da FGV.

Barreto, F. A. F. D. \& de Oliveira, L. G. S. (2001). Transição para regimes previdenciários de capitalização e seus efeitos macroeconômicos de longo prazo no Brasil. Revista Estudos Econômicos, 31(1):57 - 87.

Boucekkine, R. (1995). An alternative methodology for solving non-linear forward-looking models. Journal of Economic Dynamics and Control, 19(4):711-734.

Bouzahzah, M., la Croix, D. D., \& Docquier, F. (2002). Policy reforms and growth in computable olg economies. Journal of Dynamics and Control, 26:2093-2113. 
Figura 4 - Reforma Educacional

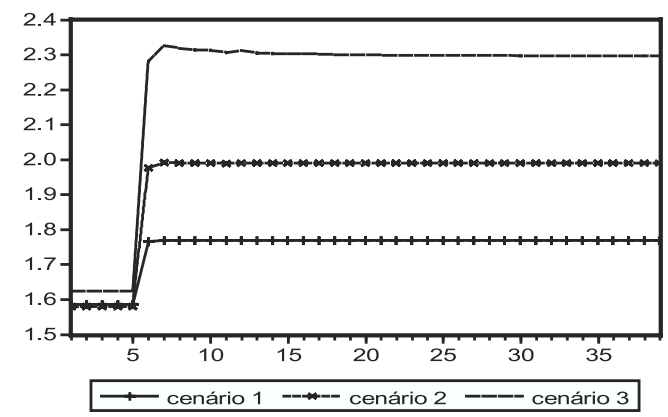

(a) Crescimento Econômico Anual

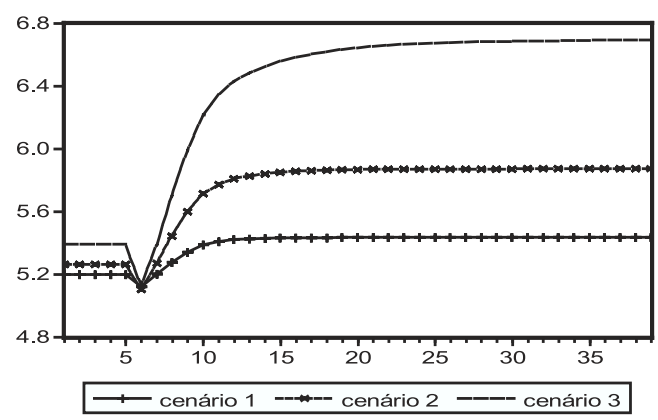

(b) Taxa de Juros Anual

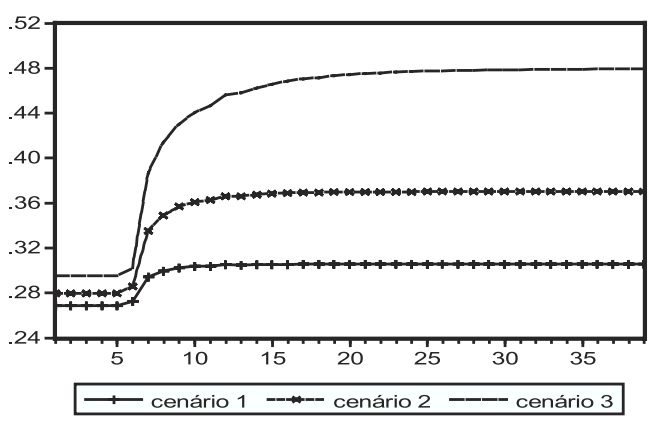

(c) Tributo sobre Salários

Casarico, A. (1998). Pension reform and economic performance under imperfect capital markets. The Economic Journal, 108(447):344-362.

Cooley, T. F. \& Prescott, E. C. (1995). Economic growth and business cycles. In Cooley, T. F., editor, Frontiers of Business Cycle Research. Princeton University Press, Princeton.

de Araújo, A. P. \& Leon, M. S. (2003). Speculative attacks on debts and optimum currency area: A welfare analysis. In Anais do XXV Encontro Brasileiro de Econometria.

de Ellery, R. \& Bugarin, M. N. S. (2003). Previdência social e bem estar no brasil. Revista Brasileira de Economia, 57(1):27-57.

Feldstein, M. (1995). The effect of consumption tax on the rate of interest. NBER Working Paper 5397.

Ferreira, P. C., Pessôa, S., \& Veloso, F. A. (2004). The evolution of international output differences (19602000): From factors to productivity. Technical Report 548, Escola de Pós-Graduação em Economia da FGV. Ensaios Econômicos da EPGE.

Fullerton, D., Shavon, J. B., \& Whalley, J. (1983). Replacing the u.s. income tax with a progressive consumption tax: A sequenced general equilibrium approach. Journal of Public Economics, 20:3-23. 
Figura 5 - Redução da Dívida Pública

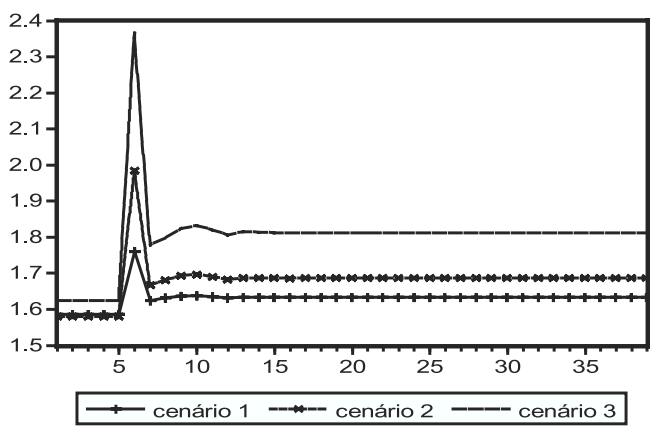

(a) Taxa de Crescimento Econômico Anual

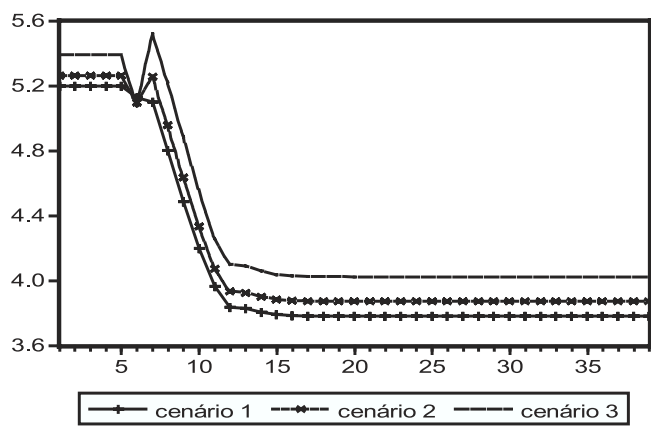

(b) Taxa de Juros Anual

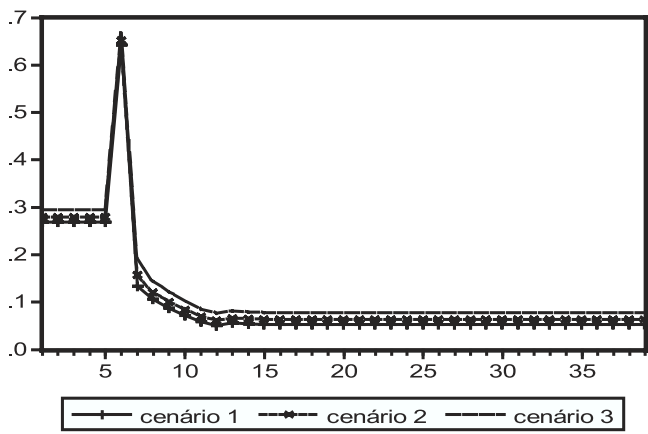

(c) Tributo sobre Salários

Gomes, V., Pessoa, S., \& Veloso, F. A. (2003). Evolução da produtividade total dos fatores na economia brasileira: uma análise comparativa. Pesquisa e Planejamento Econômico, 33(3):389-434.

Hall, R. (1996). The effect of tax reform on prices and asset values. Tax Policy and the Economy, MIT Press, Massachusetts.

Hall, R. E. \& Rabushka, A. (1996). Frontiers of Tax Reform, chapter The Flat Tax: A simple, progressive consumption tax. Hoover Institution Press, Stanford.

Heer, B. \& Trade, M. (2003). Efficiency and distribution effects of a revenue-neutral income tax reform. Journal of Macroeconomics, 25:87-107.

Issler, J. V. \& Piqueira, N. S. (2000). Estimating relative risk aversion, the discount rate, and the intertemporal elasticity of substitution in consumption for brazil using three types of utility function. Brazilian Review of Econometrics, 20(2):200-238.

Jones, L., Manuelli, R., \& Rossi, P. (1993). Optimal taxation in models of endogenous growth. Journal of Political Economy, 101(3):485-517.

Juillard, M. (1996). A program for the resolution and simulation of dynamic models with forward variables the use of a relaxation algorithm. Technical report, CEPREMAP. 
Kydland, F. \& Prescott, E. (1982). Time to built and aggregate flutuations. Econometrica, 50(6).

Lannes, J. (1999). Aspectos macroeconômicos da reforma da previdência social no Brasil: Duas análises em equilíbrio geral com restrições ao crédito. PhD thesis, Escola de Pós-Graduação em Economia da FGV.

Lin, S. (1999). Tax reform and external balance. Journal of International Money and Finance, 18:891-909.

Lucas, R. E. (1988). On the mechanics of economic development. Journal of Monetary Economics, 22:3-42.

Lucas, R. E. (1990). Supply side economics: An analytical review. Technical Report 42, Oxford.

Makdissi, P. \& Wodon, Q. (2002). Consumption dominance curves: testing for the impact of indirect tax reforms on poverty. Economics Letters, 75:227-235.

Menezes, F. \& Barreto, F. (1999). Reforma tributária no brasil: Lições de um modelo de equilíbrio geral aplicado. Revista Econômica do Nordese, 30(especial).

Mesa-Lago, C. (2002). Myth and reality of pension reform: The latin american evidence. World Development, 30(8):1309-1321.

Miles, D. (1999). Modelling the impact of demographic change upon the econommy. Economic Journal, 109:1-36.

Miranda, R. (1997). Três modelos teóricos para a previdência social. Pesquisa e Planejamento Econômico, 27(3).

Oliveira, F., Beltrão, K., \& Ferreira, M. (1997). Reforma da previdencia. Texto para Discussão do IPEA.

Orszag, P. T. \& Stiglitz, J. E. (2001). Rethinking pension reform: ten myths about social security systems. In Holzman, R. \& Stiglitz, J., editors, New ideas about social security: toward sustainable prension systems in the 21st century, pages 17-56. The World Bank, Washington.

Pechman, J. (1987). A consumption tax is not desirable for the united states. in: Walker. In Walker, C. \& Bloomfield, M., editors, The Consumption Tax. Ballinger Publishing Company.

Seidman, L. (1984). Conversion to a consumption tax: The transition in a life-cycle growth model. Journal of Political Economy, 92:247-267.

Sianesi, B. \& Reenen, J. V. (2003). The returns of education: Macroeconomis. Journal of Economic Surveys, 17(2).

Stokey, N. \& Rebelo, S. (1995). Growth effects of flat-rate taxes. Journal of Political Economy, 103:519-550.

Strulik, H. (2003). Capital tax reform, corporate finance,and economic growth and welfare. Journal of Economic Dynamics and Control, 28:595-615.

Toder, E. (1995). Comments on proposals for fundamental tax reforms. Tax Notes, pages 2003-2015. 